\title{
The truth of diabetes reversal
}

\section{Sanjay Prakash Dhangar ${ }^{1 *}$}

${ }^{1}$ SMBT Institute of Medical Sciences and Research Centre, India.

*Corresponding E-mail: sanjayamrapali18@gmail.com

\begin{abstract}
Diabetes is a real threat to health globally. It neither sees socioeconomic status nor the boundaries. There are now studies that show, at least in short term, that diabetes can be reversed. Various forms of the standard of care are available but suitability differs. Patients would surely choose reversal of their disease if given a choice. The choice can only be given if physicians are not only aware that reversal of diabetes is possible but also need to educate their patients and discuss with them various possibilities and modes of treatment. We here discuss various approaches and studies that prove that diabetes can be reversed and to what extent it is possible to reverse, the real truth about the reversal of diabetes. Apart from these approaches, further research in understanding the mechanism of diabetes reversibility and its overlapping mechanism and pathways that are lacking need to be focussed and emphasized in the future.
\end{abstract}

Keywords: diabetes, truth, metabolic syndrome, diabetes reversal, diabetes remission.

Received: 17 July, 2021

Accepted: 23 July, 2021

Published: 30 July, 2021

\section{Introduction}

Diabetes is a real threat to health globally. It neither sees socioeconomic status nor the boundaries. According to 2019 International Diabetes Federation (IDF) statistics, one in 11 adults have diabetes, one in two adults are undiagnosed with diabetes, one in five people with diabetes are above 65 years of age, $10 \%$ of the global health expenditure is spent on diabetes, one in six live births are affected by hyperglycemia in pregnancy, $84 \%$ of which have gestational diabetes, three in four people with diabetes live in low/middle income countries, over 1.1 million children and adolescents below 20 years have type I diabetes, two in three people with diabetes live in urban areas. There are approximately 463 million people with diabetes worldwide and by 2045 this will rise to 700 million [1]. India has 41 million diabetics and this number is expected to increase to 70 million by 2025 . One in six people with diabetes in the world is from India. This high number places India among the top ten countries for people with diabetes. India comes at second position after China with an estimated population of 77 million [2, 3]. Diabetes is a major public health epidemic despite recent advances in both pharmaceutical and technological treatment options.

Type 2 diabetes (T2D) has been identified as an incurable chronic disease since ancient times. What we could do maximum was the amelioration of diabetes symptoms or slowing its progression. In the past diabetes was known as chronic and irreversible but now the paradigm is shifting $[\underline{4}, \underline{5}]$.

The 2016 World Health Organization (WHO) global report on diabetes added a section on diabetes reversal hinting that it can be achieved through weight loss and calorie restriction [4]. The diabetes reversal or remission defined by an expert panel could be partial when $\mathrm{HbAlc}<6.5$ without glycaemic control medications for 1 year or complete 
when $\mathrm{HbA1c}<5.7$ without glycaemic control medications for 1 year. Prolonged remission is defined as complete remission for at least 5 years' duration. In this regard, we cannot use the term cure. Medically, the cure may be defined as restoration to good health, while remission is defined as abatement or disappearance of the signs and symptoms of a disease.

In spite of the growing evidence that reversal of diabetes is possible, achieving the same is not encouraged by our healthcare systems. In fact, it is not a goal in the diabetes guidelines. Kaiser Permanente et al., found a diabetes remission rate of $0.23 \%$ with standard of routine care [6]. Many studies show that diabetes reversal is achievable using bariatric surgery, low-calorie diets or carbohydrate restriction, etc.

Let's start with bariatric Surgery. There is unilateral improvement in glycemia following bariatric surgery [7]. It's been found to be superior to intensive T2D medical management. Currently, it is approved by 2016 WHO recommendations for adults with a body mass index $(\mathrm{BMI})>40$, or $>35 \mathrm{~kg} / \mathrm{m} 2$ with obesity-related comorbidities [8]. Glycaemic improvements occur rapidly, within hours to days, preceding weight loss. This represents the enteroendocrine responses due to the altered flow of intestinal contents like bile acid signaling and changes in microbiota and their metabolome. The basic principle of surgical treatment is restriction and intestinal malabsorption. Bariatric surgery dramatically and rapidly improves blood glucose [9] allows decreased oral hypoglycaemic medications and insulin use and effectively reverses diabetes in up to $80 \%$ of patients [10] in the short term. Long-term reversal of diabetes and true glucose homeostasis remains an uncertain point. Long-term outcomes of surgery depend on multiple factors, including the type of surgery performed, comorbidities, patient willingness for lifelong dietary change, and ongoing surveillance. The five-year outcomes of intensive medical management, sleeve gastrectomy, and Roun-en-Y gastrojejunostomy show better results in favor of Roun-en-Y gastrojejunostomy with $29-45 \%$ success. The surgical cohort also showed greater weight loss and improvements in high-density lipoproteins, triglycerides, need for insulin, and overall quality of life [11-14]. But over time, remission rates of diabetes do decline. Various studies show variable success rates of bariatric surgery from $36-83 \%$ with a follow-up from 3 years to 14 years [15-17].

Coming to low-calorie diets, there are few studies that have reported successful weight loss with decreased insulin resistance, decreased blood glucose, and reduced medication use following a low-calorie diet $[18,19]$. Weight loss persists only for the first three months in the diet-treated patients, indicating difficulty with long-term maintenance [17]. Moreover, there are micronutrient deficiencies with the use of calorie-restricted diets, and supplementation and monitoring for deficiencies should be considered whenever a low-calorie diet is started [20,21]. they demonstrated the effectiveness of calorie restriction in achieving weight loss and improved glycemic control. Remission at one year is around $11.5 \%, 9.2 \%$ at two years, and $7.3 \%$ at four years. In the LOOK AHEAD randomized trial, the remission rates achieved through intensive lifestyle intervention that included calories restriction and increased physical activity were still three to six times higher than those achieved in the diabetes support and education group [22]. Lower baseline HbAlc, a greater level of weight loss, shorter duration of diabetes diagnosis, and lack of insulin use at baseline predicted higher remission rate [23]. Overall, the evidence suggests that a low-calorie diet is effective in reversing diabetes in the short term to a maximum of two years. Few studies have suggested that physiological and metabolic adaptation of the body in response to caloric restriction may have shifted the energy balance and hormonal regulation of weight toward weight regain after initial weight loss [24, 25]. It could be due to the disturbance between the environment, lifestyle, and behavioral patterns that shift more towards the orexigenic side.

Now regarding carbohydrate-restricted diets. American Diabetes Association and the European Association for the Study of Diabetes, based on the evidence, support carbohydrate-restricted diet for control of diabetes [5,26]. Among these, a low carbohydrate diet means total carbohydrates are restricted to less than 130 grams/day and a very low 
carbohydrate diet means a ketone diet with carbohydrates as low as 20-30 grams/day. Protein consumption remains the same as in a standard diet of around $20 \%$ of the total intake. The remaining energy needs are met by fat from the diet or mobilized from the body fat stores. Carbohydrates are obtained from non-starchy vegetables with some nuts, dairy products, and fruits in limited amounts [27].

Saslow L R et al in their study found that the ketogenic diet resulted in achieving good glycaemic control and weight loss in $55 \%$ of patients [28].

Total kilocalorie intake was not significantly different between the two groups, even with moderate carbohydrate restriction. Despite equal energy intake, the low carbohydrate group lost significantly more weight and had improved glycaemic control, which indicates a potential mechanistic role for carbohydrate restriction itself. The support given was 19 classes over the 12-month period, tapering in frequency over time [29].

Another study compared a low-carbohydrate diet aimed at inducing nutritional ketosis in patients with type 2 diabetes mellitus, with usual care in type 2 diabetes mellitus patients [30]. At one year, the HbA1c decreased by $1.3 \%$ in the first group, with $60 \%$ achieving an HbAlc below $6.5 \%$ without hypoglycaemic medication. Cardiovascular risk factors also showed significant improvement [31]. The retention rate was $83 \%$ one year and 74 $\%$ at two years, supporting the sustainability of this dietary intervention for diabetes reversal. Weight loss was $10 \%$ at 2-years. The use of very-low-carbohydrate diets for diabetes reversal shows promising results but the lack of longer-term follow-up studies remains a limitation. Follow-up is limited to two years only. Therefore, longer-term studies are required to determine the sustainability of the results of metabolic changes.

Tripathi P et al., in their study of reversal of metabolic syndrome, used a polyvalent program comprising whole food plant-based diet, assorted exercises, and lifestyle modification schedule [32]. With this polyvalent program, they were able to reverse diabetes and the associated metabolic syndrome. With a similar program, they were able to achieve the reversal of diabetic nephropathy in short term [33]. But they also lack long-term results over sustenance and maintenance. Moreover, the side effects of macronutrients restriction and the whole plant-based diet were mentioned.

Paramesh Shamanna et al., used Twin Health's Precision Health platform combining Internet-of-Things (IoT) Sensors, Machine Learning (ML), and Medical Science to improve human metabolic health. Their technologyenabled precision nutrition which is a combination of macro, micro, and biota nutrients, along with serial HbAlc evaluation was used for reversal of diabetes. The rates of diabetes reversal in their study were $10 \%, 75 \%$, and $41 \%$ in $\mathrm{HbA1c}$ ranges HbA1c of $>9.5,8.1-9.5$, and 6.5-8, respectively. HbA1c was reduced by $1.9(8.8 \pm 2.23$ to $6.9 \pm$ 1.07). But their study had a limited dataset with a short duration of follow-up [34].

Another similar study by Paramesh Shamanna et al., showed that adherence for 3 months to the twin precise nutrition program resulted in patients achieving a 1.9\%-point decrease in HbAlc, 6.1\% drop in weight, 56.9\% reduction in insulin resistance, a significant decline in blood glucose below the range, and, the elimination of diabetes medication uses in most patients. This study is again a short-term study [35].

To summarise, evidence is now available to suggest that diabetes reversal is possible. In this editorial, we provide various methods that have shown successful reversal of type 2 diabetes. Among these, bariatric surgery is the most effective with good results and prolonged remission rates. The main hindrance for bariatric surgery being the surgical complications and the high treatment cost. Apart from this modification of complete lifestyle after the surgery remains a challenge. Bariatric surgery is far more than merely a restrictive and/or malabsorptive procedure. There are large shifts in the signaling of bile acid in the lumen of the small intestine, the gut sensing the nutrients, and changes in the microbiota community. There is basic and translational science research going on to identify the 
role of these various hormones and metabolites.

Low carbohydrate diet and carbohydrate-restricted diet approaches are good for reversing diabetes in the short term (2-3 years). But long-term maintenance of remission of diabetes is still a problem with these approaches. The longterm adherence to these diets remains an obstacle. The patients need proper education and optimal support for longterm behavioral change.

The polyvalent program by Tripathi et al., comprising whole plant-based diet, lifestyle modifications, behavioral modification, and proper patient education created a proper channel for the patients but long-term adherence to this approach and long-term data for remission are lacking.

Twin Health's Precision Health program of Paramesh Shamanna et al., used artificial intelligence to look for the changes made by food intake in a diabetic person. They correlated the changes of inflammation made by food/various diets that helped them control/reverse diabetes. This probably helped in understanding the mechanism of diabetes reversal. The long-term adherence and long-term data are lack in these studies.

Further research in understanding the mechanism of diabetes reversibility and its overlapping mechanism and pathways that are lacking need to be focussed and emphasized.

\section{Conclusion}

The epidemic of diabetes is causing many lives to suffer. Various forms of the standard of care are available but suitability differs. But patients would surely choose reversal of their disease if given a choice. The choice can only be given if physicians are not only aware that reversal of diabetes is possible but also need to educate their patients and discuss with them various possibilities and modes of treatment.

\section{DECLARATIONS}

\section{Funding}

This research did not receive any specific grant from funding agencies in the public, commercial, or not-for-profit sectors.

\section{Conflicts of Interest}

The authors declared no potential conflicts of interest with respect to the research, authorship, and/or publication of this article.

\section{REFERENCES}

[1] IDF Diabetes Atlas. 9th ed. International Diabetes Federation; Brussels, Belgium, 2019. International Diabetes Federation.

[2] Kannan, Ramya. "India is home to 77 million diabetics, second highest in the world". The Hindu. ISSN 0971-751X.

[3] Geoffrey Migiro. "Countries By Percentage Of World Population". WorldAtlas.

[4] World Health Organization Global Report on Diabetes. Available online: https://www.who.int/diabetes/publications/grd-2016/en/.

[5] Davies M.J., D’Alessio D.A., Fradkin J., Kernan W.N., Mathieu C., Mingrone G., Rossing P., Tsapas A., Wexler D.J., Buse J.B. Management of hyperglycemia in Type 2 diabetes, 2018. A consensus report by the American Diabetes Association (ADA) and the European Association for the Study of Diabetes (EASD) Diabetes Care. 2018; 41:2669-2701.

[6] Karter A.J., Nundy S., Parker M.M., Moffet H.H., Huang E.S. Incidence of remission in adults with Type 2 diabetes: 
The Diabetes \& Aging Study. Diabetes Care. 2014;37:3188-3195.

[7] Steven S., Carey P.E., Small P.K., Taylor R. Reversal of Type 2 diabetes after bariatric surgery is determined by the degree of achieved weight loss in both short- and long-duration diabetes. Diabet Med. 2015; 32:47-53.

[8] Rubino F., Nathan D., Eckel R.H., Schauer P.R., Alberti K.G., Zimmet P.Z., Del Prato S., Ji L., Sadikot S.M., Herman W.H., et al. Delegates of the 2nd Diabetes Surgery Summit. Metabolic surgery in the treatment algorithm for type 2 diabetes: A joint statement by International Diabetes Organizations. Diabetes Care. 2016; 39:861-877.

[9] Rubino F., Gagner M. Potential of surgery for curing type 2 diabetes mellitus. Ann. Surg. 2002;236:554-559

[10] Cohen R., Caravatto P.P., Correa J.L., Noujaim P., Petry T.Z., Salles J.E., Schiavon C.A. Glycemic control after stomach-sparing duodenal-jejunal bypass surgery in diabetic patients with low body mass index. Surg. Obes. Relat. Dis. 2012; 8:375-380.

[11] Purnell J.Q., Selzer F., Wahed A.S., Pender J., Pories W., Pomp A., Dakin G., Mitchell J., Garcia L., Staten M.A., et al. Type 2 Diabetes Remission Rates After Laparoscopic Gastric Bypass and Gastric Banding: Results of the Longitudinal Assessment of Bariatric Surgery Study. Diabetes Care. 2016; 39:1101-1107

[12] Schauer P.R., Kashyap S.R., Wolski K., Brethauer S.A., Kirwan J.P., Pothier C.E., Thomas S., Abood B., Nissen S.E., Bhatt D.L. Bariatric surgery versus medical therapy in obese patients with diabetes. N. Engl. J. Med. 2012; 366:1567-1576

[13] Kashyap S.R., Bhatt D.L., Wolski K., Watanabe R.M., Abdul-Ghani M., Abood B., Pothier C.E., Brethauer S., Nissen S., Gupta M., et al. Metabolic effects of bariatric surgery in patients with moderate obesity and type 2 diabetes: Analysis of a randomized control trial comparing surgery with intensive medical treatment. Diabetes Care. 2013; 36:2175-2182.

[14] Schauer P.R., Bhatt D.L., Kirwan J.P., Wolski K., Aminian A., Brethauer S.A., Navaneethan S.D., Singh R.P., Pothier C.E., Nissen S.E., et al. Bariatric surgery versus intensive medical therapy for diabetes: 5-year outcomes. N. Engl. J. Med. 2017; 376:641-651.

[15] Eisenbarg D., Azagury D.E., Ghiassi S., Grover B.T., Ki J.J. ASMBS position statement on postprandial hyperinsulinemic hypoglycemia after bariatric surgery. Surg. Obes. Relat. Dis. 2017; 13:371-378.

[16] Pories W.J., Swanson M.S., MacDonald K.G., Long S.B., Morris P.G., Brown B.M., Barakat H.A., de Ramon R.A., Israel G., Dolezal J.M. Who would have thought it? An operation proves to be the most effective therapy for adultonset diabetes mellitus. Ann. Surg. 1995; 222:339-350.

[17] Sjostrom L., Lindroos A.K., Peltonen M., Torgerson J., Bouchard C., Carlsson B., Dahlgren S., Larsson B., Narbro K., Sjostrom D., et al. Lifestyle, diabetes and cardiovascular risk factors 10 years after bariatric surgery. N. Engl. J. Med. 2004; 351:2683-2693.

[18] Bauman W.A., Schwartz E., Rose H.G., Eisenstein H.N., Johnson D.W. Early and long term effects of acute caloric deprivation in obese diabetic patients. Am. J. Med. 1988; 85:38-46.

[19] Hughes T.A., Gwynne J.T., Switzer B.R., Herbst C., White G. Effects of caloric restriction and weight loss on glycemic control, insulin release and resistance, and atherosclerotic risk in obese patients with type II diabetes mellitus. Am. J. Med. 1984; 77:7-17.

[20] Damms-Machado A., Weser G., Bischoff SC. Micronutrient deficiency in obese subjects undergoing low calorie diet. Nutr. J. 2012:11.

[21] Gardner C.D., Kim S., Bersamin A., Dopler-Nelson M., Otten J., Oelrich B., Cherin R. Micronutrient quality of weight-loss diets that focus on macronutrients: Results from the A to Z study. Am. J. Clin. Nutr. 2010; 92:304-312.

[22] Ryan D.H., Espeland M.A., Foster G.D., Haffner S.M., Hubbard V.S., Johnson K.C., Kahn S.E., Knowler W.C., Yanovski S.Z., Look AHEAD Research Group Look AHEAD (Action for Health in Diabetes): Design and methods for a clinical trial of weight loss for the prevention of cardiovascular disease in type 2 diabetes. Control Clin Trials. $2003 ; 24: 610-628$.

[23] Gregg E.W., Chen H., Wagenknecht L.E., Clark J.M., Delahanty L.M., Bantle J., Pownall H.J., Johnson K.C., Safford M.M., Kitabchi A.E., et al. Association of an intensive lifestyle intervention with remission of type 2 diabetes. JAMA. 2012;308:2489-2496.

[24] Fothergill E., Guo J., Howard L., Kerns J.C., Knuth N.D., Brychta R., Chen K.Y., Skarulis M.C., Walter M., 
Walter P.J., et al. Persistent metabolic adaptation 6 years after "The Biggest Loser" competition. Obesity. 2016; 24:1612-1619.

[25] Greenway F.L. Physiological adaptations to weight loss and factors favouring weight regain. Int. J. Obes. 2015; 39:1188-1196.

[26] American Diabetes Association Summary of Revisions: Standards of Medical Care in Diabetes-2019. Diabetes Care. 2019; 42:S4-S6.

[27] Westman E.C., Feinman R.D., Mavropoulos J.C., Vernon M.C., Volek J.S., Wortman J.A., Yancy W.S., Phinney S.D. Low carbohydrate nutrition and metabolism. Am. J. Clin. Nutr. 2007; 86:276-284.

[28] Saslow L.R., Mason A.E., Kim S., Goldman V., Ploutz-Snyder R., Bayandorian H., Daubenmier J., Hecht F.M., Moskowitz J.T. An online intervention comparing a very low-carbohydrate ketogenic diet and lifestyle recommendations versus a plate method diet in overweight individuals with type 2 diabetes: A randomized controlled trial. J. Med. Int. Res. 2017; 19:e36. doi: 10.2196/jmir.5806.

[29] Saslow L.R., Daubenmier J.J., Moskowitz J.T., Kim S., Murphy E.J., Phinney S.D., Ploutz-Snyder R., Goldman V., Cox R.M., Mason A.E., et al. Twelve-month outcomes of a randomized trial of a moderate-carbohydrate versus very low-carbohydrate diet in overweight adults with type 2 diabetes mellitus or prediabetes. Nutr. Diabetes. 2017; 7:304.

[30] Hallberg S.J., McKenzie A.L., Williams P.T., Bhanpuri N.H., Peters A.L., Campbell W.W., Hazbun T.L., Volk B.M., McCarter J.P., Phinney S.D., et al. Effectiveness and safety of a novel care model for the management of type 2 diabetes at 1 year: An open-label, non-randomized, controlled study. Diabetes Ther. 2018; 9:583-612.

[31] Bhanpuri N.H., Hallberg S.J., Williams P.T., McKenzie A.L., Ballard K.D., Campbell W.W., McCarter J.P., Phinney S.D., Volek J.S. Cardiovascular disease risk factor responses to a type 2 diabetes care model including nutritional ketosis induced by sustained carbohydrate restriction at one year: An open label, non-randomized, controlled study. Cardiovasc. Diabetol. 2018; 17:56.

[32] Pramod Tripathi, Maithili Paranjape and Mamata Hiremath. Reversal of Metabolic Syndrome with Freedom From Diabetes (FFD) Protocol: Elixir Endocrinology 122 (2018) 52217-52219.

[33] Pramod Tripathi, Mamata Hiremath, AnaghaVyawahare, AnayDudhbhate. Extent of Diabetic Nephropathy Reversal in Type 2 Diabetes Mellitus Patients by following the Freedom from Diabetes: Indian Journal of Public Health Research \& Development, August 2020, Vol. 11(8); 97-105.

[34] Paramesh Shamanna, Suresh Damodharan, BanshiSaboo, Rajeev Chawla, Jahangir Mohammed, Maluk Mohamed \& Mohamad Thajudeen. Utilising internet of things and artificial intelligence to enable twin precision treatment for reversal of type 2 Diabetes: Endocrine Abstracts (2020) 70 AEP1027 | DOI: 10.1530/endoabs.70.AEP1027.

[35] Paramesh Shamanna, Banshi Saboo, Suresh Damodharan, Jahangir Mohammed, Maluk Mohamed, Terrence Poon, Nathan Kleinman, and Mohamed Thajudeen. Reducing HbA1c in Type 2 Diabetes Using Digital Twin Technology-Enabled Precision Nutrition: A Retrospective Analysis: Diabetes Ther. 2020 Nov; 11(11): 27032714. 